\title{
A Micro Structure POF Relative Humidity Sensor Modified With Agarose Based on Surface Plasmon Resonance and Evanescent Wave Loss
}

\author{
Yanjun HU, Abdul GHAFFAR, Yulong HOU, Wenyi LIU*, Fei LI, and Jun WANG \\ Science and Technology on Electronic Test \& Measurement Laboratory, North University of China, Taiyuan 030051, \\ China \\ *Corresponding author: Wenyi LIUＩ-mail: liuwenyi@nuc.edu.cn
}

\begin{abstract}
A novel high sensitivity relative humidity $(\mathrm{RH})$ sensor was proposed by using micro structure plastic optical fiber (POF) based on the surface plasmon resonance (SPR) effect and the evanescent wave (EW) loss. The micro structure was fabricated on the POF and coated with a gold layer and agarose, adopting the sputtering and dip-coating technique. These construction effects on the attenuation of power caused by the SPR effect and the EW loss were used to perform RH detections. The agarose's different refractive indexes (RIs) caused fluctuations in the transmission power when the humidity increased. The demonstrated experimental results showed that the proposed sensor achieved a linear response from $20 \% \mathrm{RH}$ to $80 \% \mathrm{RH}$ with a high sensitivity of $0.595 \mu \mathrm{W} / \%$. The proposed sensor had the advantages of fast response and recovery. Furthermore, the temperature dependence and the repeatability test of the sensor were also performed.
\end{abstract}

Keywords: SPR; evanescent wave loss; agarose; RH sensor

Citation: Yanjun HU, Abdul GHAFFAR, Yulong HOU, Wenyi LIU, Fei LI, and Jun WANG, "A Micro Structure POF Relative Humidity Sensor Modified With Agarose Based on Surface Plasmon Resonance and Evanescent Wave Loss," Photonic Sensors, 2021, 11(4): 392-401.

\section{Introduction}

Relative humidity (RH) attracts lots of attention, from agriculture, medicine, and other fields [1-3]. Fiber optic RH sensors have been researched widely due to the advantages of miniaturization and electromagnetic immunity.

According to the sensor's working principle, fiber optic RH sensors are categorized into gratings, interferometers, modal interferometers, loss mode resonances (LMRs), and optical absorption [4]. Gratings have unique spectral characteristics, combined with different hydrophilic materials, which increase the system's stability and sensitivity [5-9]. However, these sensors are cross-sensitive to temperature and strain, limiting the measurement accuracy. Model interference RH sensors such as photonic crystal fiber (PCF) [10], multi-segment splicing [11], and tapered fiber [12] have attracted attention owing to special structures. However, this type of fiber has the disadvantages of high price and low robustness, which increases the difficulty of sensor fabrication and system construction. The LMR occurs to the fiber and the coating, and the dielectric constant of the coating is required to be positive [4]. Many materials such as $\mathrm{SnO}_{2}[13,14]$,

Received: 25 March 2020 / Revised: 1 August 2020

(C) The Author(s) 2020. This article is published with open access at Springerlink.com

DOI: $10.1007 / \mathrm{s} 13320-020-0603-4$

Article type: Regular 
$\mathrm{TiO}_{2}[15]$, polycyclic aromatic hydrocarbon $(\mathrm{PAH})$ [16], poly acrylic acid (PAA) [2], and some others can be applied. The sensors of LMR have good linearity, but the resonance width is large and the sensitivity is not high. Optical absorption mainly utilizes the absorption of light intensity by the sensitive material. This type of sensor has a simple structure, but the output waveform is vulnerable to interference from ambient light, and the RH linearity range is small $[17,18]$.

Detection of surface plasmon resonance (SPR) is another principle for fiber optic humidity sensors which happens in a metallic layer (such as gold and silver) and a dielectric layer (analytes). For the fiber optic SPR sensors, wavelength interrogation is widely used because it can locate the position of the resonance wavelength. However, the testing equipment is expensive, and the accuracy of the results is affected by the full width at half maximum (FWHM) [19-23]. For the single-mode fiber (SMF), the FWHM makes the resonance wavelength easy to be recognized, but there is no change in the angle of incidence inside, so special treatment is needed to change the reflection angle [24-26]. It increases the difficulty of processing. Multimode fiber such as plastic optical fiber (POF) can solve the incident angle problem. However, the FWHM of the POF is too large, causing a read deviation of the resonant wavelength. Therefore, it is important to choose the correct and appropriate interrogation method. Intensity interrogation is independent of FWHM and focuses on changes in intensity. Furthermore, the experimental setup is simple and the output waveform is more intuitive.

In this work, the micro structure fiber was made by grinding paper, a $50 \mathrm{~nm}$ gold layer was sputtered on the surface of the micro structure fiber, and $0.5 \mathrm{wt} \%$ agarose was finally applied as a sensitive material. The SPR effect was excited at the interface of the gold and agarose layer, which was particularly sensitive to changes in the environment. Compared with the optical absorption sensors, the combined effect of the SPR effect and evanescent wave (EW) loss significantly increased the sensor's sensitivity, and the proposed sensor could increase the transmission power and reduce the external light interference. Moreover, the micro structure of the proposed sensor was simpler than those of the gratings and interferometers sensors. The response to the sensor at different RHs was tested by using intensity interrogation. The experimental results showed that the sensitivity was up to $0.595 \mu \mathrm{W} / \% \mathrm{RH}$ at $20 \% \mathrm{RH}-80 \% \mathrm{RH}$, and the sensitivity was $0.305 \mu \mathrm{W} / \% \mathrm{RH}$ at $80 \% \mathrm{RH}-$ $90 \% \mathrm{RH}$. The above experiments proved that the proposed sensor had the high sensitivity and good linearity.

\section{Working principle}

The light propagates in the optical fiber due to total internal reflection when the angle of the incident light is larger than the critical angle. The propagation mode characterizes the propagation of light rays inside the fiber. The number of propagation modes is characterized by $V$-number (normalized frequency) which is expressed as (1) $[27,28]$. The penetration depth $(d)$ can be expressed as (2) [29].

$$
\begin{gathered}
V=\frac{2 \pi r}{\lambda} \sqrt{n_{\mathrm{co}}-n_{\mathrm{cl}}} \\
d=\frac{\lambda}{2 \pi \sqrt{n_{\mathrm{co}}^{2} \sin ^{2} \theta_{c}-n_{\mathrm{cl}}^{2}}}
\end{gathered}
$$

where $r$ is the core-radius of fiber, $\lambda$ represents the wavelength of the light source, $n_{\mathrm{co}}$ and $n_{\mathrm{cl}}$ are the refractive indexes (RIs) of the core and cladding, respectively, and $\theta_{c}$ is the incidence angle of the light at the core-cladding interface.

The higher-order guided modes in the micro structure area are filtered out. Consequently, in the tapered region, the evanescent field intensity increases. The effect of the penetration depth $\left(d^{\prime}\right)$ and $V$-number are expressed as (3) [27] and (4) [29]. 


$$
\begin{gathered}
V_{t}=\frac{2 \pi r_{t}(z)}{\lambda} \sqrt{n_{\mathrm{co}}-n_{\mathrm{en}}} \\
d^{\prime}=\frac{\lambda}{2 \pi \sqrt{n_{\mathrm{co}}^{2} \sin ^{2} \theta_{\mathrm{int}}-n_{\mathrm{en}}^{2}}}
\end{gathered}
$$

where $z$ represents the radius of the micro structure fiber, $r_{t}(z)$ represents a function of $z$, $\theta_{\text {int }}$ is the incident angle at the core-external environment interface, and $n_{\mathrm{en}}$ is the external environmental RI for instance air or humidity condition. When RI of the environment changes from air to moisture, the values of the $V$-number $\left(V_{t}\right)$ and penetration depth $\left(d^{\prime}\right)$ will be changed, causing the change in the output power.

Figure 1 shows the schematic diagram of the proposed sensor structure. When the gold layer is attached to the surface of the micro structure POF, it will stimulate the surface plasma wave (SPW) on the surface of the gold film. When the propagation constant of the incident light is equal to that of the SPW, the strong absorption of the light occurs, causing the loss of the transmission power. The resonance condition is expressed in (5) [30,31].

$$
K_{0} n_{\mathrm{co}} \sin \theta=K_{0} \sqrt{\left(\frac{\varepsilon_{\mathrm{mr}} \times n_{s}^{2}}{\varepsilon_{\mathrm{mr}}+n_{s}^{2}}\right)} ; K_{0}=\frac{2 \pi}{\lambda}
$$

where $\varepsilon_{\mathrm{mr}}$ represents the real part of the metal dielectric constant, $\theta$ is the incident angle at the core-gold layer interface, and $n_{s}$ represents the refractive index of the sensing layer [29]. The SPR effect is heavily sensitive to environmental changes and is suitable for the measurement of different parameters. According to the characteristics of the multimode fiber, the countless modes exist inside the core, with different propagation angles. Therefore, when the propagation angle is the same as the incident angle $\theta$ in (5), the SPR effect can be excited.

The evanescent wave loss is the sensitive principle of the sensor when the micro structure fiber has no gold layer. After the gold layer is attached to the surface of the fiber, the evanescent field is absorbed by the SPR effect, and the transmission power is lost. When the SPR effect occurs at the resonance wavelength, the loss reaches its maximum. For the proposed sensor, the transmission power was affected by the SPR effect under the attenuated total reflection (ATR) effect and the evanescent wave (EW) loss, and the intensity change of the proposed sensor was greater than that under the influence of EW loss.

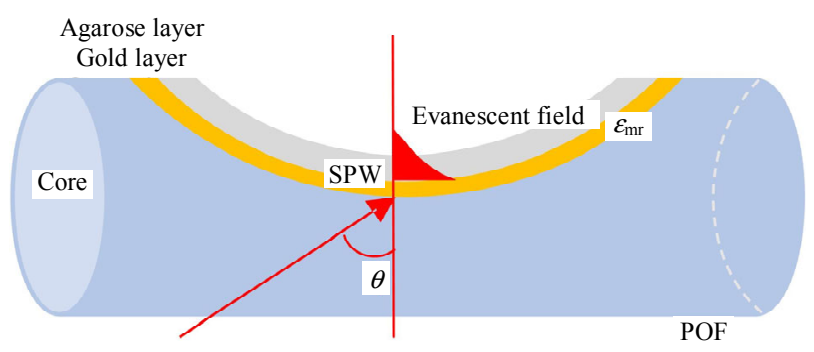

Fig. 1 Schematic diagram of the proposed sensor.

A wavelength interrogation system was set up to realize the humidity sensor's sensitivity mechanism, including the SPR effect. The experimental setup consisted of an optical spectral analyzer (OSA) with a wavelength of $300 \mathrm{~nm}-2600 \mathrm{~nm}$ (AQ6374, YOKOGAWA Test \& Measurement, Tokyo, Japan) and a halogen light source with a wavelength of $350 \mathrm{~nm}-1750 \mathrm{~nm}$ (SLS201, Thorlabs, Newton, NJ, USA). Figure 2 shows the transmission spectra at different humidity levels. As shown in Fig. 2, the SPR effect occurs from $650 \mathrm{~nm}$ to $690 \mathrm{~nm}$, and the wavelength of $660 \mathrm{~nm}$ is included in it. Therefore, it can be proved that the wavelength of $660 \mathrm{~nm}$ is related to the SPR effect's excitation. Besides, it can also be concluded from Fig. 2 that the wavelength of

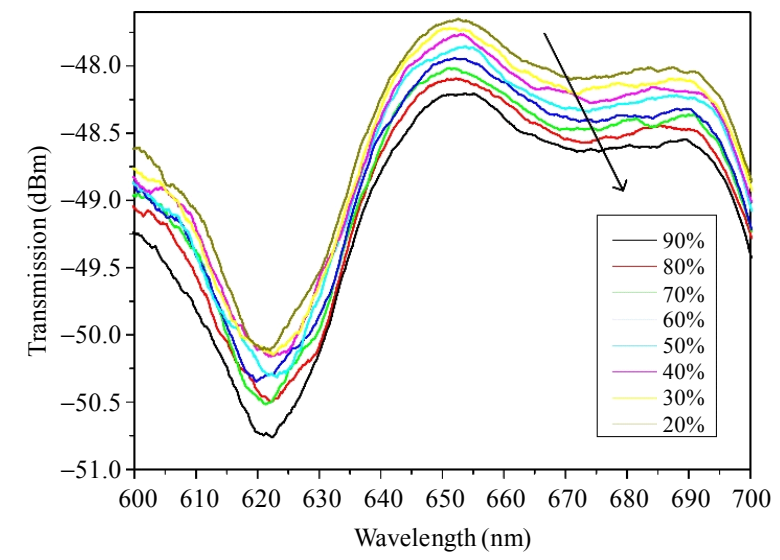

Fig. 2 Transmission spectrums of the proposed sensor. 
the proposed sensor does not change significantly but requires data smoothing, which will increase the system processing time and affect the real-time performance of the sensor. Therefore, the intensity modulation is selected.

\section{Experiment}

\subsection{Sensor fabrication}

A POF (Mitsubishi, Tokyo, Japan, SK40) was taken with a length of $30 \mathrm{~cm}$. The complete processing method and parameters of the POF are illustrated in Fig. 3(a). A polishing tool was made by using a 3-dimensional (3D) printer (A8, JG AURORA, Shenzhen, China). The parameters of the micro structure fiber are shown in Fig. 3(b). After the tool was made, the POF was fixed in the structure's groove, and the sandpaper of 2000 mesh was fixed on the desktop. The processing was completed when the depth of the polished fiber was the same as that of the groove. The distance of the groove was $2 \mathrm{~cm}$, and the depth of the groove was $400 \mu \mathrm{m}$. After the micro structure fiber was washed with deionized water and dried, it was placed in a magnetron sputtering apparatus (NJ08057, Denton, USA), a gold target was selected, and the relevant processing parameters were set according to the thickness of the sputtering. The thickness of the sputtering was $50 \mathrm{~nm}$. The parameters of the magnetron sputtering process are shown in Table 1.

Batumalay et al. [32] studied the effect of agarose concentration on humidity sensitivity and proved that the highest sensitivity was at $0.5 \mathrm{wt} \%$ concentration, so this concentration was used in this work. The $0.5 \mathrm{wt} \%$ agarose gel was configured through agarose powder (Sigma Aldrich, St. Louis, A6013, USA) and distilled water. The agarose powder and distilled water were heated up at $65^{\circ} \mathrm{C}$ with a magnetic stirrer (MS-H280-Pro, Dragon lab, Beijing, China). Then, the dip-coating technique was used, the fiber was immersed in the hot agarose (a)

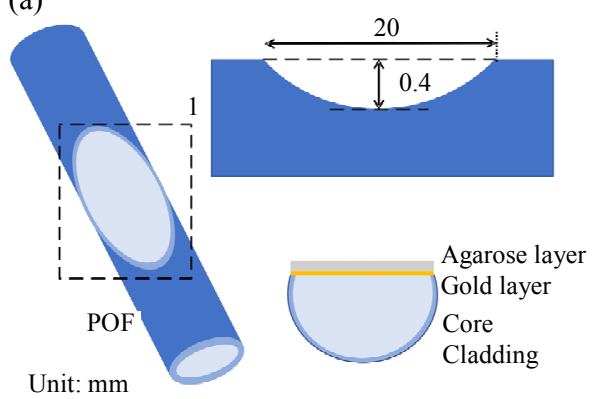

(b)

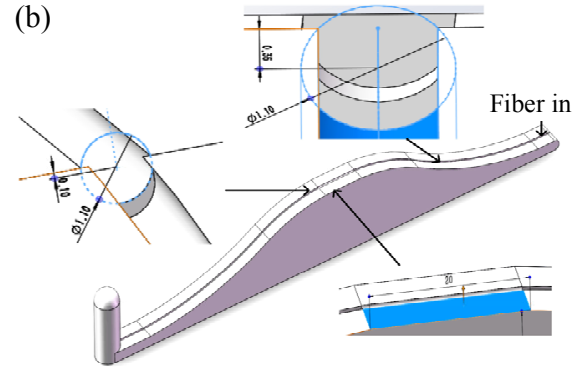

Fig. 3 Parameters of (a) the micro structure fiber and (b) the polishing tool.

Table 1 Parameters of the magnetron sputtering process.

\begin{tabular}{ccccc}
\hline Power $(\mathrm{W})$ & $\begin{array}{c}\text { Time } \\
\text { (s) }\end{array}$ & $\begin{array}{c}\text { Vacuum } \\
\text { degree (torr) }\end{array}$ & $\begin{array}{c}\text { Argon gas flow } \\
\text { velocity (sccm) }\end{array}$ & $\begin{array}{c}\text { Rotate } \\
\text { speed (\%) }\end{array}$ \\
\hline 500 & 20 & $5 \mathrm{e}^{-6}$ & 14 & 30 \\
\hline
\end{tabular}

solution, the fiber was taken out after $1 \mathrm{~s}$, and the fiber was immersed in the solution again after $1 \mathrm{~s}$. This test was repeated five times. The prepared probes were placed to dry at the room temperature for $24 \mathrm{~h}$, and the humidity experiment was carried out.

\subsection{Experimental setup}

The LED light was selected as a light source with a wavelength of $660 \mathrm{~nm}$. When the input wavelength was in a band where the SPR effect and evanescent wave loss coexisted, the change in the sensor's output intensity was better than those at other wavelengths. Moreover, the POF was in the visible light range, and the optical fiber output loss was small. The cost of the intensity interrogation system was small. The light emitting diode (LED) light sources used were stable and did not require optical isolation to replace laser sources that were susceptible to echo interference. 
Figure 4 shows the experimental structure of the proposed humidity measurement sensor, and the image of the sensing fiber probe is shown in Fig. 4(b). The experimental setup consisted of a light source (M660F1) with $660 \mathrm{~nm}$ wavelength, an optical power meter (PM100USB, Thorlabs), and a humidity chamber. A thermos-hygrometer (AH8008, China) and a designed probe were placed inside the humidity chamber. Thermos-hygrometer was used for the reference. The initial temperature of the humidity chamber was set to about $25{ }^{\circ} \mathrm{C}$. The humidity varied from $20 \%$ to $90 \%$, and the response of the proposed sensor was investigated. The external light was easy for coupling in the POF due to the large diameter of the fiber. The fiber was covered with a black jacket to shield the external light to avoid the interference of the visible light [33]. The host computer with software (Thorlabs optical power meter, Thorlabs, Newton, NJ, USA) recorded the changes in the transmission waveform changes and values.

(a)

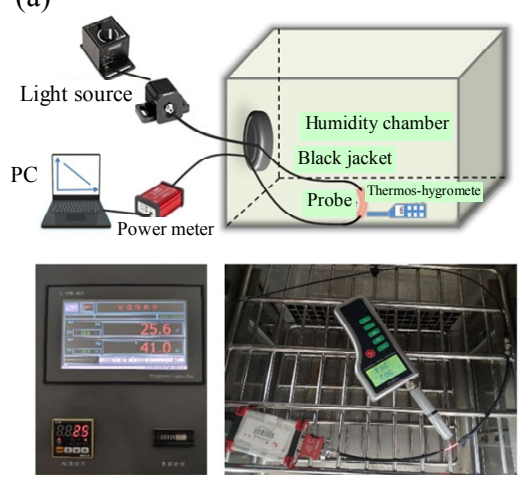

Fig. 4 Illustration of the experiment: (a) experimental setup of the proposed sensor and (b) the picture of the POF probe.

\section{Results and discussion}

\subsection{Using agarose \& without agarose}

The experimental equipment was connected, as shown in Fig. 4(a). The humidity chamber temperature was set to $25^{\circ} \mathrm{C}$, and the change in the output light intensity corresponded to the change in humidity. The effect of agarose on the $\mathrm{RH}$ response to the proposed sensor was also investigated in the experiment. Figure 5 shows the achieved results. In Fig. 5(a), the slope of the response curve with agarose is significantly higher than the slope of the response curve without agarose. It indicated that the sensor with the agarose sensor was more sensitive to humidity than that without agarose. This loss occurring in transmitted power was due to the effect of the SPR effect and evanescent wave (EW) loss, and its intensity change was more significant than that under the influence of EW loss. Therefore, combining the SPR effect and EW loss could effectively increase the sensitivity of the sensor.

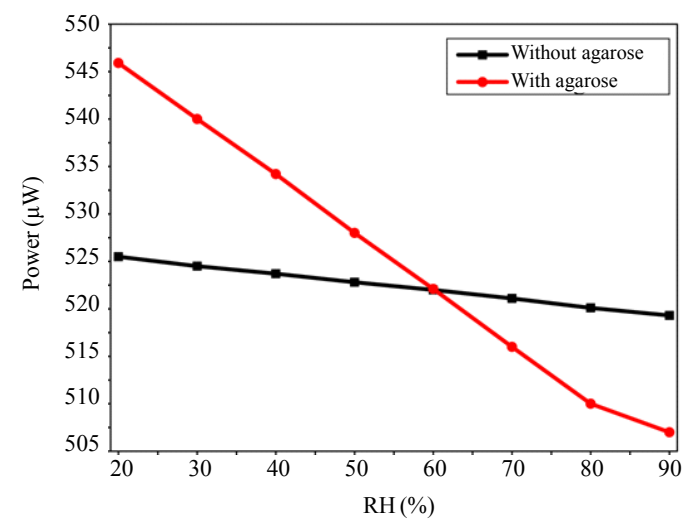

(a)

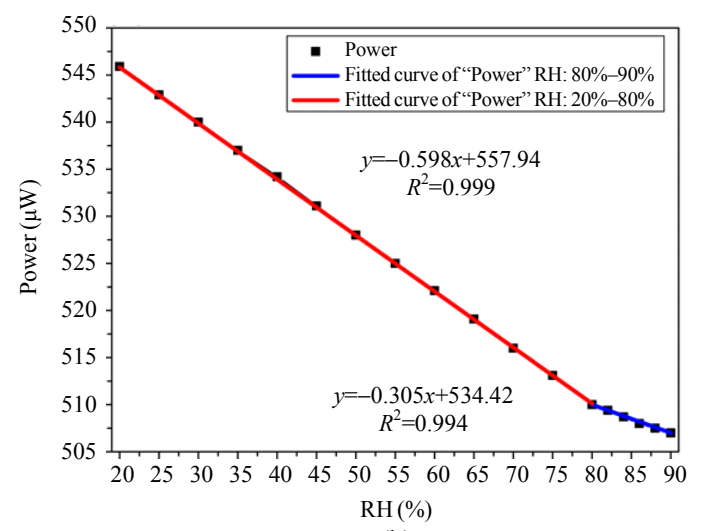

(b)

Fig. 5 Humidity detection results of the proposed sensor: (a) proposed sensor's transmission power response using agarose and without agarose and (b) linear fitting curve of the sensor coated agarose.

A further test was performed to determine the relationship between the humidity and output power, and the results are shown in Fig. 5(b). It could be proved that the linearity range of the sensor was $20 \%-80 \%$ and $80 \% \mathrm{RH}-90 \% \mathrm{RH}$ in Fig. 5(b), the sensor sensitivity with agarose was $0.595 \mu \mathrm{W} / \% \mathrm{RH}$ 
when $\mathrm{RH}$ raised from $20 \%$ to $80 \%$, and the sensitivity was $0.305 \mu \mathrm{W} / \% \mathrm{RH}$ when $\mathrm{RH}$ changed from $80 \%$ to $90 \%$. The determination coefficients $\left(R^{2}\right)$ of the two curves were 0.999 and 0.994 .

Table 2 summarizes the sensitivity and detection range of the designed sensor compared with the other RH measurement sensors based on intensity interrogation. As shown in Table 2, the proposed sensor presents good linearity and wide dynamic humidity operating range with the humidity variation because the agarose is a high humidity-sensitivity material in a wide range of relative humidity.

Table 2 Comparison of the sensitivity and detection range for RH sensors.

\begin{tabular}{ccccc}
\hline Reference & Fiber structure & $\begin{array}{c}\text { Sensing } \\
\text { material }\end{array}$ & Sensitivity & $\begin{array}{c}\text { Detection } \\
\text { range (\%) }\end{array}$ \\
\hline [18] & $\begin{array}{c}\text { Side-polished } \\
\text { fiber }\end{array}$ & $\begin{array}{c}\text { Reduced } \\
\text { grapheme } \\
\text { oxide }\end{array}$ & $0.31 \mathrm{~dB} / \% \mathrm{RH}$ & $75-95$ \\
{$[34]$} & Micro fiber & WS2 & $0.196 \mathrm{~dB} / \% \mathrm{RH}$ & $37-90$ \\
{$[35]$} & $\begin{array}{c}\text { Mach-Zehnder } \\
\text { interferometer }\end{array}$ & $\begin{array}{c}\text { Grapheme } \\
\text { oxide/PVA }\end{array}$ & $0.193 \mathrm{~dB} / \% \mathrm{RH}$ & $25-80$ \\
{$[36]$} & Core-offset fiber & $\begin{array}{c}\text { Grapheme } \\
\text { oxide }\end{array}$ & $0.104 \mathrm{~dB} / \% \mathrm{RH}$ & $30-60$ \\
{$[37]$} & Micro-bending & Rhodamine & $1.6 \mathrm{mV} / \% \mathrm{RH} \&$ & $10-45 \&$ \\
tapered POF & $6 \mathrm{G}$ & $3.4 \mathrm{mV} / \% \mathrm{RH}$ & $45-90$ \\
[38] & Tapered POF & Agarose & $0.0228 \mathrm{mV} / \% \mathrm{RH}$ & $50-85$ \\
& & & $0.595 \mu \mathrm{W} / \% \mathrm{RH}$ & \\
This & Micro structure & Au and & $(20 \%-80 \%)$ & $20-80 \&$ \\
paper & POF & agarose & $0.305 \mu \mathrm{W} / \% \mathrm{RH}$ & $80-90$ \\
& & & $(80 \%-90 \%)$ & \\
\hline
\end{tabular}

4.2 Repeatability, stability, reversibility, and consistency

To measure the repeatability of the designed humidity measurement sensor, RH was increased and decreased from $20 \%$ to $90 \%$ five times. Figure 6(a) shows the results of this experiment. As can be seen from Fig. 6(a), the output waveform of the proposed sensor was virtually the same, indicating that the proposed sensor had the good repeatability. In order to test the stability of the sensor, the output power changed at $20 \%$ and $90 \%$, and humidities were tested, as shown in Fig. 6(b). It was observed that the output power had hardly changed over a while, indicating that the sensor had a good stability.
The transmission waveform shown in Fig. 6(b) had fewer fluctuations. It was caused by the instability of the instrument output and was an inevitable factor, but the output curve of the repetitive test was consistent.

The reversibility is a necessary factor to evaluate a sensor. As given in Fig. 6(c), the transmission power of the proposed sensor was recorded when the humidity was cycled between $20 \%$ and $90 \%$. The maximum difference was $0.2 \mu \mathrm{W}$ which was smaller compared with the transmission power. The final results proved that the designed sensor had a good reversibility. To verify the consistency of the humidity sensor, three samples (Samples 1, 2, and 3) were processed for the humidity test. The experimental results are shown in Fig.6(d). As can be seen from Fig. 6(d), when the humidity was $20 \%-90 \%$, the three samples' output curve trends were the same, indicating that the humidity sensor had a good consistency.

\subsection{Temperature dependence}

RI of the POF changes with temperature due to the thermal coefficient of the fiber. Changes in the refractive index of the sensitive material agarose at different temperatures also cause the differences in the transmission power. As a result, the temperature dependence was investigated. The temperatures of the humidity chamber were set at $25^{\circ} \mathrm{C}, 35^{\circ} \mathrm{C}$, and $45^{\circ} \mathrm{C}$, respectively. The experimental results are shown in Fig. 7. As the temperature increased, the transmission power gradually decreased. It indicated that the proposed sensor was independent of temperature, while there was almost no change in the slope of the output curve. The change of temperature did not affect the linearity and the sensitivity of the proposed sensor. Temperature compensation could be performed by introducing a thermometer when the ambient temperature changed.

Due to fiber material's influence, the POF sensor varied greatly at different temperatures, which was a 
limitation of the sensor. Temperature compensation will be a future research direction. At present, the

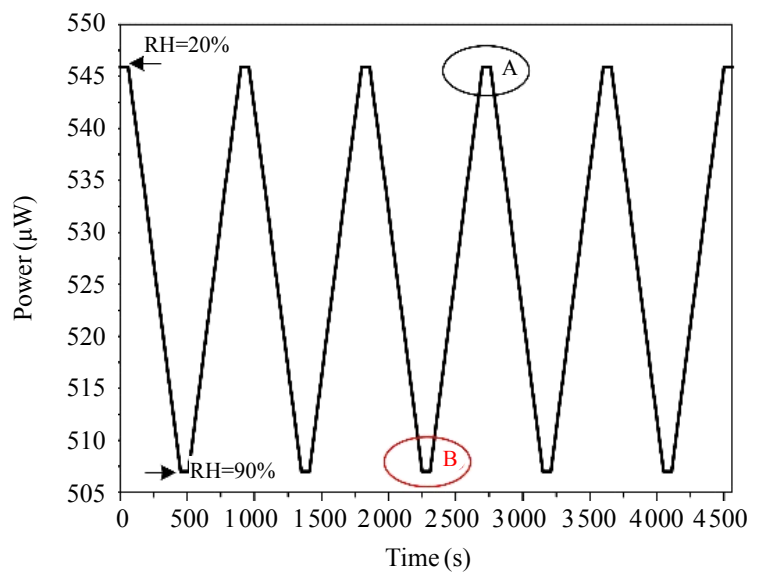

(a)

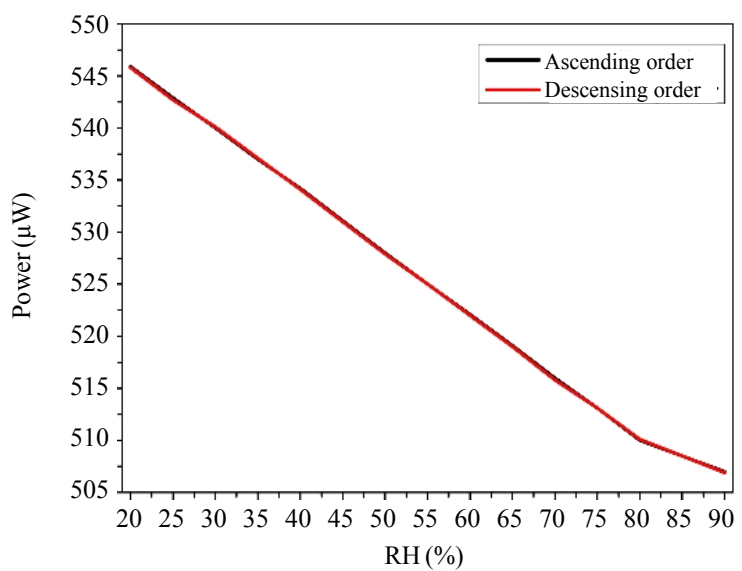

(c) proposed sensor was only suitable for the environment at a constant temperature.

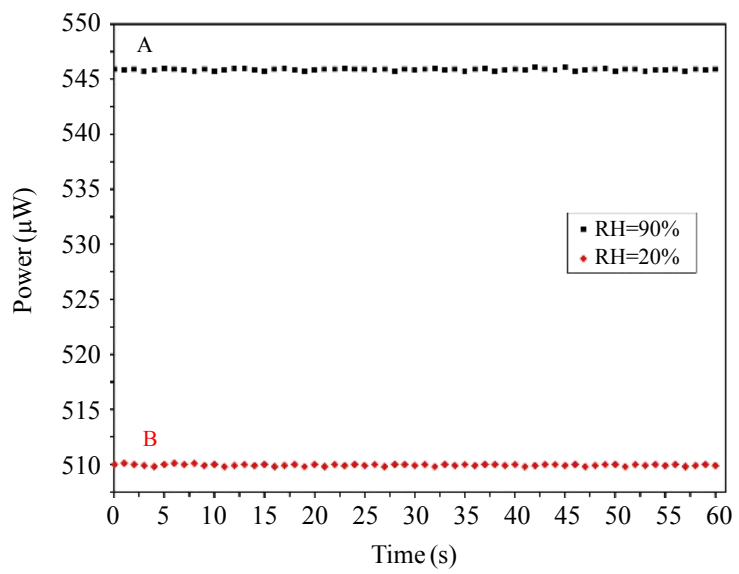

(b)

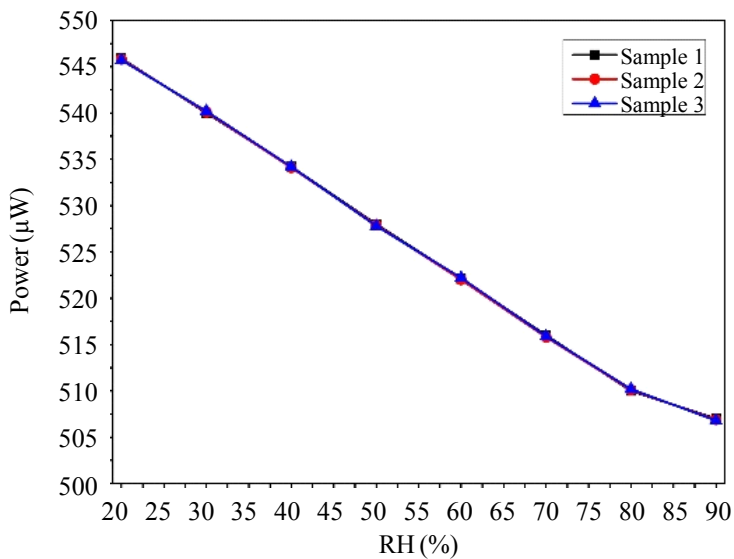

(d)

Fig. 6 Proposed sensor tests of (a) the repeatability, (b) the stability, (c) the reversibility, and (d) the consistency.

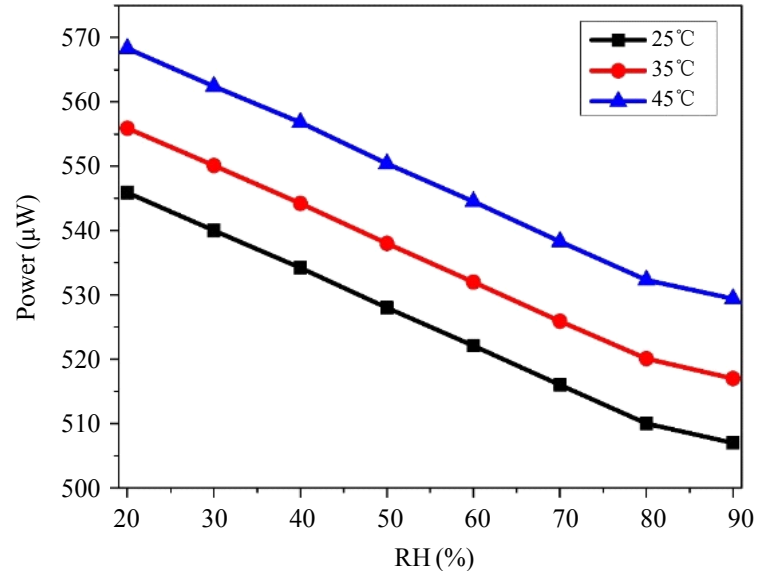

Fig. 7 Measured the transmission powers of the proposed sensor at $25^{\circ} \mathrm{C}, 35^{\circ} \mathrm{C}$, and $45^{\circ} \mathrm{C}$, respectively.

\subsection{Response and recovery time}

The proposed sensor was placed at room temperature and exhaled for $1 \mathrm{~s}$ to obtain the dynamical response times. The detection setup is shown in Fig. 8(a). The temperature was $25^{\circ} \mathrm{C}$ and the ambient humidity during the experiment was $40 \% \mathrm{RH}$. The sensor response to the human breath was measured. The result is shown in Fig. 8(b). The time interval of software data collection was $0.1 \mathrm{~s}$, so the time of 8 collection points was $0.8 \mathrm{~s}(800 \mathrm{~ms})$, and the 40 collection points represented $4 \mathrm{~s}$. So the output power immediately responded in $800 \mathrm{~ms}$, while the value of the output power returned to the 
initial level after $4 \mathrm{~s}$. The results proved that the sensor could respond quickly to human breathing, indicating that the sensor is suitable for biological or industrial environments with strict humidity response requirements.
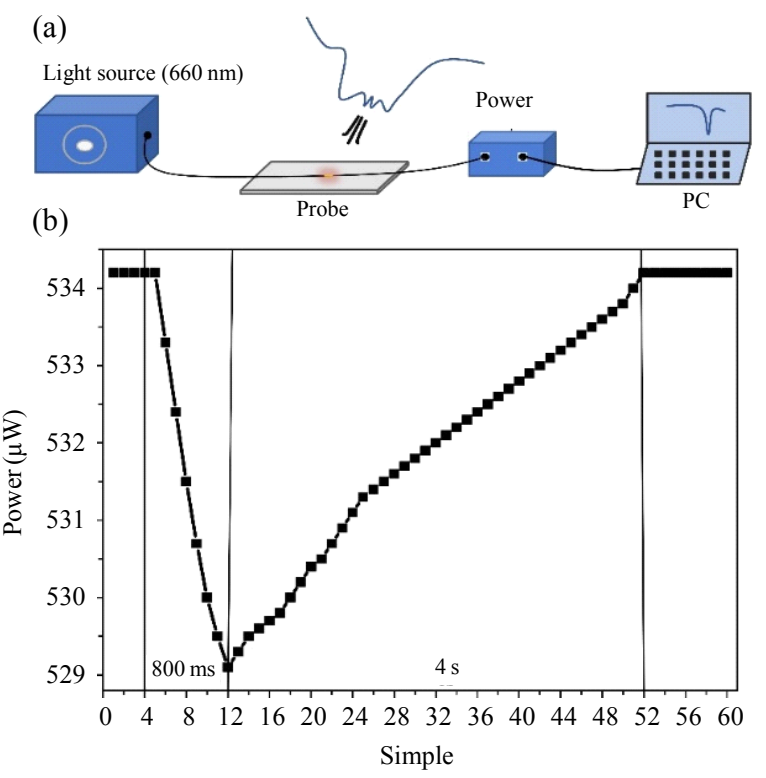

Fig. 8 Results of response and recovery time: (a) schematic diagram of the detection setup and (b) dynamical response of the proposed sensor to the breath.

\section{Conclusions}

A cost-effective and high-sensitivity RH sensor based on the SPR effect has been proposed. The proposed sensor was constructed by functionalizing a gold-coated micro structure POF with agarose which was inexpensive and simple. The RH testing was performed by intensity interrogation. The response repeatability and reversibility of the sensor to RH were studied in this work. The experimental results showed that when the RH changed from $20 \%$ to $80 \%$, the sensor had a good output linearity with a sensitivity of $0.595 \mu \mathrm{W} / \%$. The proposed sensor had a wide linear range with the high sensitivity which was suitable for environmental humidity detection.

\section{Acknowledgment}

This work was funded by the Fund of Shanxi "1331" Project Key Subject Construction; National Science Foundation of Shanxi Province,
China (Grant No. 201701D121065). This work is accomplished at the Science and Technology on Electronic Test \& Measurement Laboratory, North University of China. The authors wish to thank the members of the Center for Micron and Nano research at the North University of China for the provision of technical support during the work.

Open Access This article is distributed under the terms of the Creative Commons Attribution 4.0 International License (http://creativecommons.org/licenses/by/4.0/), which permits unrestricted use, distribution, and reproduction in any medium, provided you give appropriate credit to the original author(s) and the source, provide a link to the Creative Commons license, and indicate if changes were made.

\section{References}

[1] L. Alwis, T. Sun, and K. T. V. Grattan, "Optical fibre-based sensor technology for humidity and moisture measurement: review of recent progress," Measurement, 2013, 46(10): 4052-4074.

[2] F. U. Hernandez, S. P. Morgan, B. R. Hayes-Gill, D. Harvey, W. Kinnear, A. Norris, et al., "Characterization and use of a fiber optic sensor based on $\mathrm{PAH} / \mathrm{SiO}_{2}$ film for humidity sensing in ventilator care equipment," IEEE Transactions on Biomedical Engineering, 2016, 63(9): 1985-1992.

[3] A. Barrias, J. R. Casas, and S. Villalba, "A review of distributed optical fiber sensors for civil engineering applications," Sensors, 2016, 16(5): 748-1-748-35.

[4] J. Ascorbe, J. M. Corres, F. J. Arregui, and I. R. Matias, "Recent developments in fiber optics humidity sensors," Sensors, 2017, 17(4): 893-1-893-23.

[5] A. Urrutia, J. Goicoechea, A. L. Ricchiuti, D. Barrera, S. Sales, and F. J. Arregui, "Simultaneous measurement of humidity and temperature based on a partially coated optical fiber long period grating," Sensors and Actuators B: Chemical, 2016, 227(5): 135-141.

[6] Y. Q. Wang, C. Y. Shen, W. M. Lou, F. Y. Shentu, C. Zhong, X. Y. Dong, et al., "Fiber optic relative humidity sensor based on the tilted fiber Bragg grating coated with graphene oxide," Applied Physics Letters, 2016, 109(3): 031107-1031107-5.

[7] G. Yan, Y. Liang, E. H. Lee, and S. He, "Novel Knob-integrated fiber Bragg grating sensor with polyvinyl alcohol coating for simultaneous relative humidity and temperature measurement," Optics 
Express, 2015, 23(12): 15624-15634.

[8] Z. W. Xie, H. T. Yan, Y. F. Li, and X. Y. Zhao, "A humidity fiber sensor based on both end-sides of a fiber Bragg grating coated with polyimide," Optical Fiber Technology, 2020, 57: 102220.

[9] H. T. Yan, Q. Liu, Y. Ming, W. Luo, Y. Chen, and Y. Q. Lu, "Metallic grating on a D-shaped fiber for refractive index sensing," IEEE Photonics Journal, 2013, 5(5): 4800706-4800706.

[10] S. Zheng, Y. Zhu, and S. Krishnaswamy, "Fiber humidity sensors with high sensitivity and selectivity based on interior nanofilm-coated photonic crystal fiber long-period gratings," Sensors and Actuators B: Chemical, 2013, 176: 264-274.

[11] R. Gao, D. F. Lu, J. Cheng, Y. Jiang, L. Jiang, and Z. M. Qi, "Humidity sensor based on power leakage at resonance wavelengths of a hollow core fiber coated with reduced graphene oxide," Sensors and Actuators B: Chemical, 2016, 222: 618-624.

[12] M. R. K. Soltanian, A. S. Sharbirin, M. M. Ariannejad, I. S. Amiri, R. M. De La Rue, G. Brambilla, et al., "Variable waist-diameter Mach-Zehnder tapered-fiber interferometer as humidity and temperature sensor," IEEE Sensors Journal, 2016, 16(15): 5987-5992.

[13] J. Ascorbe, J. M. Corres, I. R. Matias, and F. J. Arregui, "High sensitivity humidity sensor based on cladding-etched optical fiber and lossy mode resonances," Sensors and Actuators B: Chemical, 2016, 233: 7-16.

[14] H. Chen, Z. Gu, and K. Gao, "Humidity sensor based on cascaded chirped long-period fiber gratings coated with $\mathrm{TiO}_{2} / \mathrm{SnO}_{2}$ composite films," Sensors and Actuators B: Chemical, 2014, 196: 18-22.

[15] C. R. Zamarreño, M. Hernaez, P. Sanchez, I. Del Villar, I. R. Matias, and F. J. Arregui, "Optical fiber humidity sensor based on lossy mode resonances supported by $\mathrm{TiO}_{2} / \mathrm{PSS}$ coatings," Procedia Engineering, 2011, 25: 1385-1388.

[16] P. Sanchez, C. R. Zamarreno, M. Hernaez, I. Del Villar, I. R. Matias, and F. J. Arregui, "Considerations for lossy-mode resonance-based optical fiber sensor," IEEE Sensors Journal, 2012, 13(4): 1167-1171.

[17] S. K. Khijwania, K. L. Srinivasan, and J. P. Singh, "An evanescent-wave optical fiber relative humidity sensor with enhanced sensitivity," Sensors and Actuators B: Chemical, 2005, 104(2): 217-222.

[18] Y. Xiao, J. Zhang, X. Cai, S. Z. Tan, J. H. Yu, H. H. $\mathrm{Lu}$, et al., "Reduced graphene oxide for fiber-optic humidity sensing," Optics Express, 2014, 22(25): 31555-31567.

[19] A. M. Shrivastav, S. K. Mishra, and B. D. Gupta, "Fiber optic SPR sensor for the detection of melamine using molecular imprinting," Sensors and Actuators B: Chemical, 2015, 212: 404-410.
[20] N. Cennamo, G. D’Agostino, M. Pesavento, and L. Zeni, "High selectivity and sensitivity sensor based on MIP and SPR in tapered plastic optical fibers for the detection of L-nicotine," Sensors and Actuators B: Chemical, 2014, 191: 529-536.

[21] N. Cennamo, M. Pesavento, L. Lunelli, L. Vanzetti, C. Pederzolli, L. Zeni, et al., "An easy way to realize SPR aptasensor: a multimode plastic optical fiber platform for cancer biomarkers detection," Talanta, 2015, 140: 88-95.

[22] R. Verma and B. D. Gupta, "A novel approach for simultaneous sensing of urea and glucose by SPR based optical fiber multianalyte sensor," Analyst, 2014, 139(6): 1449-1455.

[23] Z. K. Fan, S. G. Li, Q. Liu, G. W. An, H. L. Chen, J. S. Li, et al., "High sensitivity of refractive index sensor based on analyte-filled photonic crystal fiber with surface plasmon resonance," IEEE Photonics Journal, 2015, 7(3): 1-9.

[24] Z. H. Liu, Y. Wei, Y. Zhang, C. L. Liu, Y. X. Zhang, E. M. Zhao, et al., "Distributed fiber surface plasmon resonance sensor based on the incident angle adjusting method," Optics Letters, 2015, 40(19): 4452-4455.

[25] Y. Wei, C. L. Liu, Y. H. Zhang, Y. X. Luo, X. F. Nie, Z. H. Liu, et al., "Multi-channel SPR sensor based on the cascade application of the single-mode and multimode optical fiber," Optics Communications, 2017, 390: 82-87.

[26] Z. H. Liu, Y. Wang, Y. Zhang, Y. S. Wang, E. M. Zhao, Y. X. Zhang, et al., "A multi-channel fiber SPR sensor based on TDM technology," Sensors and Actuators B: Chemical, 2016, 226: 326-331.

[27] A. Leung, K. Rijal, P. M. Shankar, and R. Mutharasan, "Effects of geometry on transmission and sensing potential of tapered fiber sensors," Biosensors and Bioelectronics, 2006, 21(12): 2202-2209.

[28] D. J. Feng, M. S. Zhang, G. X. Liu, X. L. Liu, and D. F. Jia, "D-shaped plastic optical fiber sensor for testing refractive index," IEEE Sensors Journal, 2014, 14(5): 1673-1676.

[29] I. Puspita, F. I. Husada, and A. M. Hatta, "Detection of lard adulteration in olive oil by using tapered plastic optical fiber (POF) spectroscopy," in Third International Seminar on Photonics, Optics, and Its Applications (ISPhOA 2018), Indonesia, 2019, pp. $110440 \mathrm{H}$

[30] Y. S. Dwivedi, A. K. Sharma, and B. D. Gupta, "Influence of design parameters on the performance of a surface plasmon sensor based fiber optic sensor," Plasmonics, 2008, 3(2-3): 79-86.

[31] M. Kanso, S. Cuenot, and G. Louarn, "Sensitivity of optical fiber sensor based on surface plasmon resonance: modeling and experiments," Plasmonics, 
2008, 3(2-3): 49-57.

[32] M. Batumalay, S. W. Harun, F. Ahmad, R. M. Nor, N. R. Zulkepely, and H. Ahmad, "Study of a fiber optic humidity sensor based on agarose gel," Journal of Modern Optics, 2014, 61(3): 244-248.

[33] A. Ghaffar, Y. L. Hou, W. Y. Liu, F. A. Dharejo, H. X. Zhang, P. G. Jia, et al., "Two-dimensional displacement optical fiber sensor based on macro-bending effect," Optics \& Laser Technology, 2019, 120: 105688 .

[34]H. Y. Guan, K. Xia, C. Y. Chen, Y. H. Luo, J. Y. Tang, H. H. Lu, et al., "Tungsten disulfide wrapped on micro fiber for enhanced humidity sensing," Optical Materials Express, 2017, 7(5): 1686-1696.

[35] Y. Wang, C. Shen, W. Lou, and F. Shentu, "Fiber optic humidity sensor based on the graphene oxide/PVA composite film," Optics Communications, 2016, 372: 229-234.

[36] S. Liu, H. Meng, S. Deng, Z. Wei, F. Wang, and C. Tan, "Fiber humidity sensor based on a graphenecoated core-offset Mach-Zehnder interferometer," IEEE Sensors Letters, 2018, 2(3): 1-4.

[37] Z. Guo, F. H. Chu, J. Y. Fan, Z. Zhang, Z. L. Bian, G. F. Li, et al., "Study of macro-bending biconical tapered plastic optical fiber for relative humidity sensing," Sensor Review, 2019, 39(3): 352-357.

[38] M. Batumalay, S. W. Harun, N. Irawati, H. Ahmad, and H. Arof, "A study of relative humidity fiber-optic sensors," IEEE Sensors Journal, 2014, 15(3): 1945-1950. 